\title{
Hypoalbuminemia as a risk factor for thromboembolic events in inflammatory bowel disease inpatients
}

\author{
Marcello Rabello Imbrizi ${ }^{1}$, Daniela Oliveira Magro ${ }^{2}$, Tirzah de Mendonça Lopes Secundo ${ }^{1}$, Marlone Cunha-Silva ${ }^{1}$, \\ Paulo Gustavo Kotze ${ }^{3}$, Ciro Garcia Montes ${ }^{1}$, Jazon Romilson de Souza Almeida ${ }^{1}$, Virgínia Lúcia Ribeiro Cabral ${ }^{1}$ \\ ${ }^{1}$ Gastroenterology Unit, Department of Internal Medicine and ${ }^{2}$ Colorectal Surgery Unit, Department of Surgery, University of Campinas \\ (UNICAMP), Campinas; ${ }^{3}$ Colorectal Surgery Unit, IBD Outpatients Clinic, Cajuru University Hospital, Catholic University of Parana (PUCPR), \\ Curitiba, Brazil
}

Background/Aims: Inflammatory bowel disease (IBD) are chronic entities characterized by local and systemic inflammation and may be associated with thrombosis. The aim of this study was to identify the prevalence of thromboembolic events (TEE) in hospitalized IBD patients and identify risk factors for their occurrence. Methods: This retrospective, single-center study included patients treated at a Brazilian IBD referral unit between 2004 and 2014. Patients hospitalized for more than 48 hours due to active IBD and who did not receive prophylaxis for TEE during hospitalization were included. Patients were allocated to 2 groups: those with TEE up to 30 days or at the time of hospitalization (TEE-group) and patients without TEE (control-group). Clinical and laboratory characteristics were evaluated. Results: Of 53 patients evaluated, 69,8\% with Crohn's disease (CD) and $30.2 \%$ with ulcerative colitis (UC). The prevalence of TEE 30 days before or during hospitalization was 15.1\%, with $10.8 \%$ in CD and $25 \%$ in UC. In the TEE group, mean serum albumin was $2.06 \mathrm{~g} / \mathrm{dL}$ versus $3.30 \mathrm{~g} / \mathrm{dL}$ in the control group. Patients with albumin levels below $2.95 \mathrm{~g} / \mathrm{dL}$ (43.18\%) had a higher risk of developing TEE (relative risk, 1.72; 95\% confidence interval, 1.17-2.53) $(P<0.001)$. Conclusions: Albumin levels were significantly lower in patients with TEE, and hypoalbuminemia was considered a risk factor for the development of TEE in this population. (Intest Res 2019;17:63-69)

Key Words: Crohn disease; Colitis, ulcerative; Thromboembolism; Thromboembolic prophylaxis; Serum albumin

\section{INTRODUCTION}

Inflammatory bowel disease (IBD), mainly represented by UC and CD, are characterized by chronic inflammation of the GI tract and may be associated with extraintestinal manifestations, including thromboembolic events (TEE). ${ }^{1}$

A relationship between IBD and TEE was first described in 1936 in a study involving more than 1,000 patients with IBD

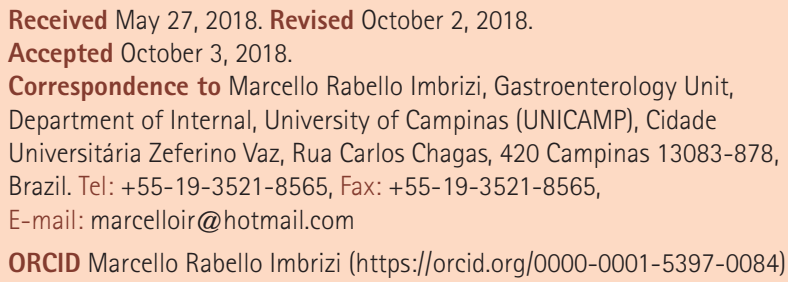

followed at the Mayo Clinic (USA), of whom 1.2\% had TEE. ${ }^{2}$ Since then, several studies have been demonstrating such association, with the incidence of TEE in this group of patients being estimated between $1.2 \%$ and $6.7 \%$, reaching $39 \%$ in studies that evaluated necropsies. Such events occur mostly at times when IBD is active or complicated by stenosis, fistulas, or abscesses. ${ }^{3,4}$

There is still no consensus between the difference in the incidence of TEE between UC or CD, although many studies show similar rates in both diseases. Irving et al. ${ }^{4}$ described, in a population-based study, an incidence of 40:10,000 people per year for CD and 50:10,000 people per year for UC. The most frequent association with UC was explained by the greater frequency of pancolonic inflammatory involvement, which is the disease extension mostly associated with TEE. ${ }^{4,5}$ 
The association between inflammation and TEE is well documented, but few studies have been able to identify risk factors for TEE in IBD. These risk factors are: an inversely proportional relationship between age at diagnosis and incidence of TEE; disease activity, whose active phase was related to vasoocclusive events; the location and extent of the disease, in which TEE appear to be more frequent in patients with CD with active colonic disease and in those with UC and extensive disease. ${ }^{6,7}$ Risk factors described in other populations were also referred to those with IBD as smoking, use of hormonal contraceptives, hyperhomocysteinemia, prolonged immobilization, infections, central venous catheters, long journeys and obesity. $^{5}$

The aim of this study was to investigate the prevalence of TEE and associated risk factors in hospitalized patients with IBD as a result of the disease.

\section{METHODS}

\section{Study Design}

A retrospective study was carried out through the analysis of the medical records of admitted patients from the IBD unit of the Hospital de Clínicas of the University of Campinas (UNICAMP), a tertiary referral center from Campinas, São Paulo, Brazil, from 2004 to 2014.

\section{Inclusion and Exclusion Criteria}

We included all inpatients for more than 48 hours, whose reasons for hospitalization were related to active or complicated IBD. Patients without a diagnosis of IBD, pregnant women, patients with hematopoietic diseases, patients with contraindication to antithrombotic prophylaxis, those with coagulopathies, and taking anticoagulant medications up to 30 days before admission were excluded.

Patients with other systemic diseases at risk for TEE such as diabetes, hypertension, dyslipidemia and metabolic syndrome, for example, were not excluded, as well as advanced age, which was not considered an exclusion criterion.

\section{Variables Analyzed}

The Harvey Bradshaw simplified index (HBI) was used to evaluate disease activity in CD and, for UC, the Truelove and Witts index was used..$^{8,9}$ Diseases were categorized according to the Montreal classification (age at diagnosis, location in the GI tract and behavior). ${ }^{6}$

All therapeutic procedures which the patients underwent, during hospitalization and previous ones, were described. The use of oral contraceptives and previous or active smoking habits were also investigated.

Body composition assessment was performed by weight and BMI $\left(\mathrm{kg} / \mathrm{m}^{2}\right)$ in the first 48 hours of hospitalization. Physical status was classified into underweight grade 1, 2, and 3 (BMI: 17-18.49, 16-16.99, and $<16 \mathrm{~kg} / \mathrm{m}^{2}$, respectively), normal weight (BMI: 18.5-24.9 kg/m²) and overweight/obesity grade 1, 2, and 3 (BMI: 25.0-29.99, 30-39, and $>39 \mathrm{~kg} / \mathrm{m}^{2}$, respectively). ${ }^{10}$ Laboratory tests collected during the same period were also analyzed: albumin (reference value [RV], 3.5-5.2 $\mathrm{g} / \mathrm{dL})$, prealbumin $(\mathrm{RV}, 20-40 \mathrm{mg} / \mathrm{dL}), \mathrm{CRP}(\mathrm{RV},<0.3 \mathrm{mg} / \mathrm{dL})$ and $\operatorname{ESR}(\mathrm{RV},<10 \mathrm{~mm} / \mathrm{hr})$.

The investigation of the TEE was performed in patients with suspicious clinical symptoms, according to the physician's discretion. TEE were defined as any venous or arterial thrombosis, clinically detectable and proven by imaging methods (Doppler ultrasonography or computerized angiotomography). Thromboembolism was considered when there were evident clinical symptoms confirmed by imaging methods (computerized angiotomography).

\section{Group Definition}

Patients were allocated into 2 groups: those with TEE up to 30 days before or during hospitalization (TEE group) and patients without TEE (control group). The period of 30 days had an arbitrary definition, as an attempt to detect patients who will develop TEE before and after admission with an adequate time frame.

\section{Statistical Analysis}

Data analyses were performed using SPSS version 20.0 (IBM Corp., Armonk, NY, USA). The results were expressed as medians and percentiles (interquartile range, $25 \%-75 \%$ ) for quantitative variables. The Mann-Whitney $U$-test (non-parametric distribution) were used to compare continuous variables between categories. Dichotomous variables were compared, between groups, using chi-square test or Fischer exact test. Receiver operating characteristic (ROC) analysis was performed to determine the cut off level of hypoalbuminemia using criterion of the distance from point (0.1). RR was calculated to identify possible risk factors for TEE. Differences with $P<0.05$ were considered statistically significant.

\section{Ethical Aspects}

The protocol was approved by the Institutional Ethics Review 
Board at University of Campinas (UNICAMP), in Campinas, Brazil, under reference number 51482015.0000.5404. The informed consent was waived.

\section{RESULTS}

\section{Demographic Characteristics}

A total of 53 hospitalizations of IBD patients who not use anti-

Table 1. Baseline Characteristics

\begin{tabular}{|c|c|c|c|}
\hline Characteristics & $\begin{array}{l}\text { TEE } \\
\text { group } \\
(n=8)\end{array}$ & $\begin{array}{c}\text { Non-TEE } \\
\text { group } \\
(n=45)\end{array}$ & $P$-value \\
\hline \multicolumn{4}{|l|}{ Sex } \\
\hline Female & $4(50)$ & $23(51)$ & 0.60 \\
\hline Male & $4(50)$ & $22(49)$ & 0.62 \\
\hline Cardiovascular diseases & $1(12)$ & $6(13)$ & 0.71 \\
\hline Hyperlipidemia & 0 & $2(4)$ & 0.72 \\
\hline Diabetes & 0 & $3(6)$ & 0.71 \\
\hline CD (Montreal classification) & $4(50)$ & $33(73)$ & $<0.001$ \\
\hline Age at diagnostic & & & 0.60 \\
\hline A1 & 0 & $5(15)$ & \\
\hline$A 2$ & $4(100)$ & $26(78)$ & \\
\hline A3 & 0 & $2(6)$ & \\
\hline Location of the disease & & & 0.62 \\
\hline L1 & 0 & 0 & \\
\hline L2 & $2(50)$ & $19(57)$ & \\
\hline L3 & 0 & $8(24)$ & \\
\hline L4 & $1(25)$ & $2(6)$ & \\
\hline $\mathrm{L} 2+\mathrm{L} 4$ & $1(25)$ & $4(12)$ & \\
\hline Disease phenotype & & & 0.41 \\
\hline B1 & $2(50)$ & $16(48)$ & \\
\hline B2 & $1(25)$ & $9(27)$ & \\
\hline B3 & $1(25)$ & $8(24)$ & \\
\hline UC (Montreal classification) & $4(50)$ & $12(27)$ & 0.07 \\
\hline Disease extention & & & 0.06 \\
\hline E1 & 0 & $2(16)$ & \\
\hline E2 & 0 & $3(25)$ & \\
\hline E3 & $4(100)$ & $7(58)$ & \\
\hline Age at diagnosis (yr) & & & 0.05 \\
\hline$<17$ & 0 & 0 & \\
\hline $17-40$ & $1(25)$ & $8(66)$ & \\
\hline$>40$ & $3(75)$ & $4(33)$ & \\
\hline
\end{tabular}

Values are presented as number (\%).

TEE, thromboembolic events. thtombotic prophylaxis during hospitalization was analyzed, 37 with CD and 16 with UC (Fig. 1). The baseline characteristics of the patients are described in detail in Table 1.

\section{Prevalence of Thromboembolic Events}

The prevalence of TEE, 30 days before or during hospitalization, was $15.1 \%(\mathrm{n}=8)$, with a significant difference between diseases, being $10.8 \%$ in CD and $25 \%$ in UC $(P=0.004)$. We observed a predominance of venous TEE (87.5\%) with 6 deep vein thrombosis (3 lower limbs, 2 pelvis, 1 abdomen), 1 pulmonary thromboembolism and only 1 arterial TEE (stroke). There was no difference in TEE according to sexes. Regarding the prevalence of TEE in relation to age, in CD there was a predominance of young adults, between 17 and 40 years of age, while in UC it was higher in adults over 40 years.

The frequency of TEE was higher in patients with colonic CD and in those with pancolitis in UC ( $75 \%$ and $100 \%$, respectively). The median hospitalization period was 13.5 days (range, 5.75-20 days) in the TEE group and 9 days (range, 2.50-14 days $)$ in the control group $(P=0.22)$. There was no significant difference in the median duration of the disease, being 2 years (range, 1-3.75 years) and 7 years (range, 1-15 years) in the TEE and control groups respectively $(P=0.26)$. From the 8 patients who presented TEE, $62.5 \%$ were diagnosed at hospital admission.

\section{Risk Factors Associated with Thromboembolic Events}

Regarding body weight, we observed that in the TEE group, BMI was lower than in the control group. There was no association between BMI and TEE, although the TEE group had more prevalence of underweight grade $1 .{ }^{10}$

ROC analysis identified an albumin levels serum $\leq 2.95 \mathrm{~g} / \mathrm{dL}$ (area under the ROC curve of $0.859, P=0.002$, sensitivity $100 \%$, specificity $72.2 \%$ [ = 1-0.278]) (Fig. 2).

Serum levels of prealbumin and albumin were lower in the TEE group, with significant association with albumin levels $(P<0.001)$. Nineteen patients $(43.18 \%)$ presented hypoalbuminemia $(\leq 2.95 \mathrm{~g} / \mathrm{dL})$ and we observed a higher risk of developing TEE in this group specifically (RR, 1.72; 95\% CI, 1.172.53) $(P<0.001)$. These data are illustrated in detail in Table 2.

When analyzing separately individuals with serum albumin $\leq 2.95 \mathrm{~g} / \mathrm{dL}$ and the other patients, we did not find a significant difference between the variables studied: increased CRP or ESR, platelet levels, prealbumin levels, BMI, the duration of the disease and active of disease index. In this group, $25 \%$ were 


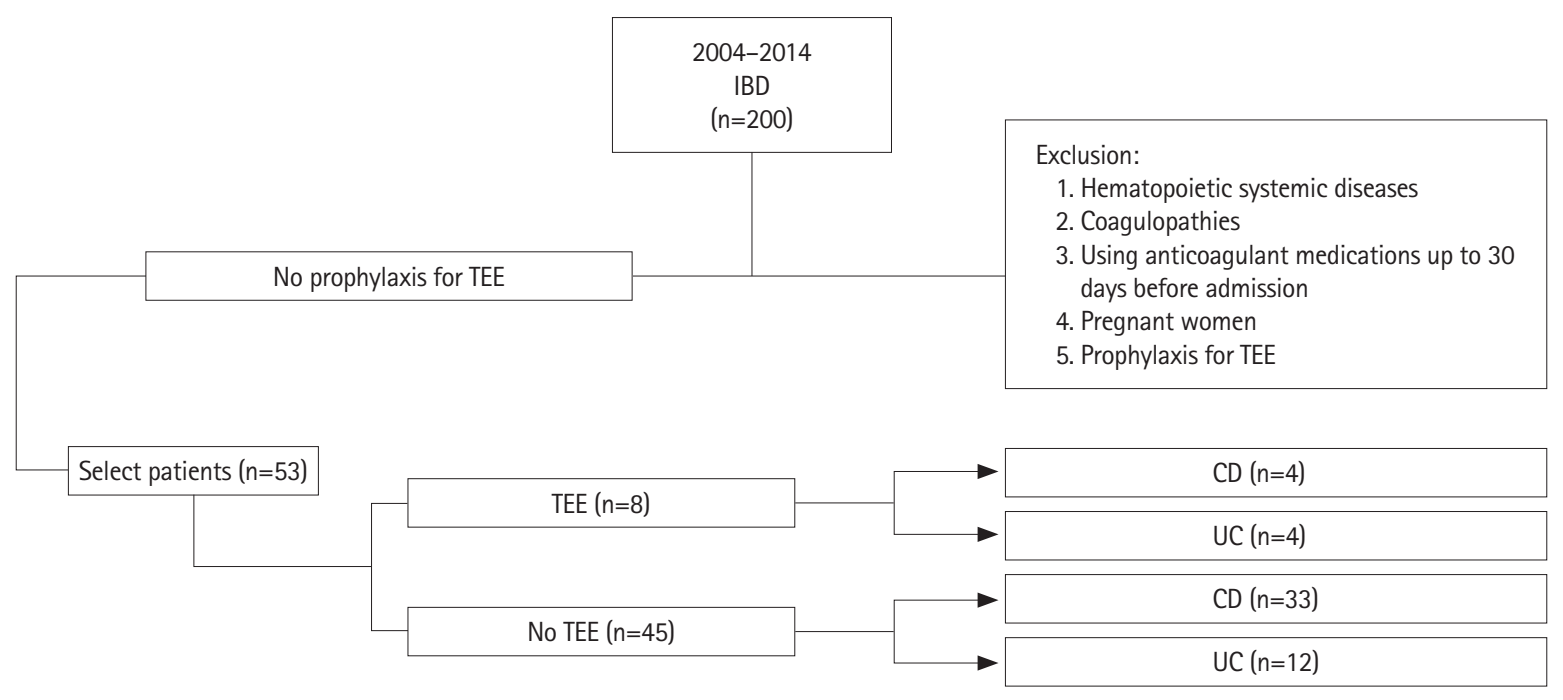

Fig. 1. Patients flowchart. TEE, thromboembolic events.

Table 2. Clinical and Laboratory Characteristics of Patients with and without TEE

\begin{tabular}{|c|c|c|c|}
\hline Variable & TEE group $(n=8)$ & Non-TEE group $(n=45)$ & $P$-value \\
\hline Length of hospital stay (day) & $13.50(5.75-20.00)$ & $9.00(2.50-14.00)$ & 0.220 \\
\hline Duration of disease (yr) & $2.00(1.00-3.75)$ & $7.00(1.00-15.00)$ & 0.260 \\
\hline Albumin (g/dL) & $2.05(1.47-2.62)$ & $3.40(2.72-3.90)$ & 0.001 \\
\hline$C D(g / d L)$ & $2.00(1.47-2.52)$ & $3.20(2.00-3.90)$ & 0.045 \\
\hline UC (g/dL) & $2.25(1.35-2.77)$ & $3.50(3.40-4.20)$ & 0.003 \\
\hline Prealbumin (mg/dL) & $10.80(7.16-21.90)$ & $13.60(7.94-29.48)$ & 0.500 \\
\hline Platelet count $\left(\times 10^{3} / \mu \mathrm{L}\right)$ & $394(278-738)$ & $309(225-433)$ & 0.490 \\
\hline BMI $\left(\mathrm{kg} / \mathrm{m}^{2}\right)$ & $20.45(16.25-21.50)$ & $20.99(18.38-23.49)$ & 0.320 \\
\hline $\mathrm{CRP}(\mathrm{mg} / \mathrm{mL})$ & $6.12(4.27-7.82)$ & $2.90(0.55-7.36)$ & 0.220 \\
\hline ESR (mm/hr) & $53.00(5.00-117.00)$ & $36.50(25.75-88.50)$ & 0.740 \\
\hline$C D^{\mathrm{a}}$ & $3(75)$ & $27(82)$ & 0.001 \\
\hline$U c^{b}$ & $4(100)$ & $11(92)$ & 0.120 \\
\hline Smoking & $2(25)$ & $2(4)$ & 0.001 \\
\hline Oral contraceptives & 0 & 0 & - \\
\hline
\end{tabular}

Values are presented as median (interquartile range) or number (\%).

${ }^{a}$ Harvey Bradshaw index (activity).

${ }^{\circ}$ Truelove and Witts (severe).

TEE, thromboembolic events.

Mann-Whitney U-test, chi-square test.

smokers (Table 3). None of them underwent prolonged immobilization, had surgeries, central venous catheters, used oral contraceptives or were submitted to hormone replacement.

\section{DISCUSSION}

Thromboembolic complications are significant causes of mor- bidity and mortality in patients with IBD. ${ }^{1}$ The objective of this study was to investigate the prevalence and risk factors for TEE in a historical cohort of a Brazilian tertiary hospital, in order to identify a risk that would increase awareness and lead to the use of thromboprophylaxis.

TEE occurred in $15 \%$ of the patients studied. This prevalence is considered high when compared to other publications. ${ }^{2,3}$ 
Table 3. Clinical and Laboratory Characteristics of Patients with and without Hypoalbuminemia

\begin{tabular}{|c|c|c|c|}
\hline \multirow{2}{*}{ Variable } & \multicolumn{2}{|c|}{ Albumin $(\mathrm{g} / \mathrm{dL})$} & \multirow{2}{*}{$P$-value } \\
\hline & $\leq 2.95(n=18)$ & $>2.95(n=26)$ & \\
\hline TEE & $8(100)$ & 0 & - \\
\hline Duration of disease (yr) & $1(0-7.50)$ & $7.50(2-14.25)$ & 0.07 \\
\hline Prealbumin (mg/dL) & $9.94(7.33-21.05)$ & $28.10(10.56-30.45)$ & 0.08 \\
\hline Platelet count $\left(\times 10^{3} / \mu \mathrm{L}\right)$ & $308(205-514)$ & $351(243-454)$ & 0.77 \\
\hline $\mathrm{BMI}\left(\mathrm{kg} / \mathrm{m}^{2}\right)$ & 19.90 (17.00-21.00) & 21.04 (18.88-23.49) & 0.11 \\
\hline $\mathrm{CRP}(\mathrm{mg} / \mathrm{mL})$ & $5.44(2.89-10.76)$ & $1.37(0.48-6.87)$ & 0.94 \\
\hline ESR (mm/hr) & $46.00(11.75-73.25)$ & $35.50(25.75-96.25)$ & 0.61 \\
\hline$C D^{a}$ & $12(66.7)$ & $13(50.0)$ & 0.84 \\
\hline$U C^{b}$ & $4(22.2)$ & $11(42.3)$ & 0.11 \\
\hline Smoking & $2(11.1)$ & $2(7.7)$ & 0.07 \\
\hline
\end{tabular}

Values are presented as number (\%) or median (interquartile range).

${ }^{a}$ Harvey Bradshaw index.

${ }^{b}$ Truelove and Witts (severe).

TEE, thromboembolic events.

Mann-Whitney U-test; chi-square test.

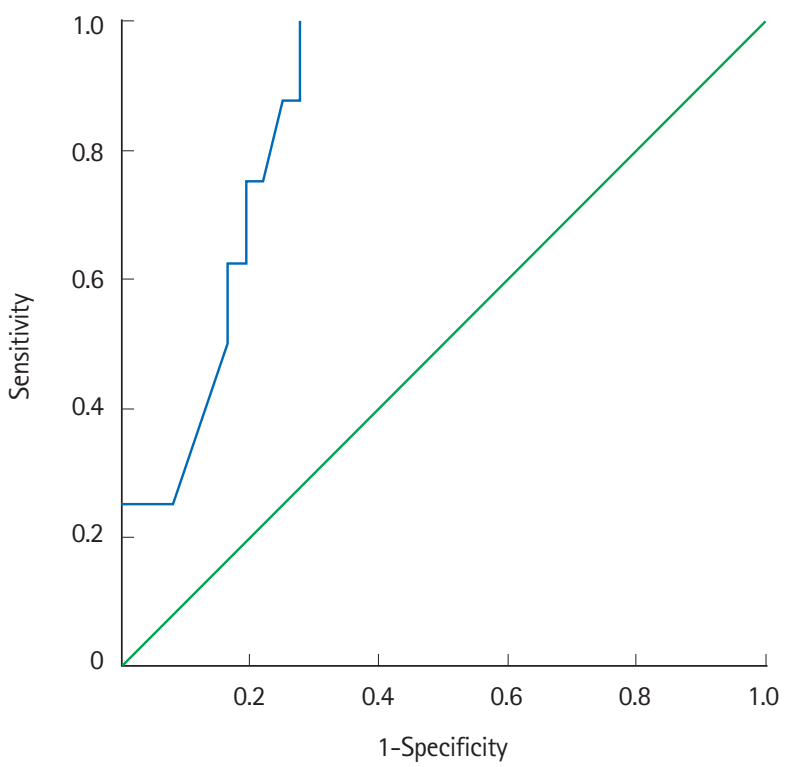

Fig. 2. Receiver operating characteristic $(\mathrm{ROC})$ analysis. Area under the ROC curve of $0.859(P=0.002 ; 95 \% \mathrm{Cl}, 0.75-0.97$; sensitivity $100 \%$; specificity $72.2 \%[=1-0.278])$.

This can be explained by all patients belonging to a tertiary IBD unit, probably presenting a more complex, active and severe disease. We also selected patients who, due to physicians' discretion, did not receive antithrombotic prophylaxis during the hospitalization period, and this can represent a selection bias in our study. ${ }^{2,4}$ As the TEE investigation was only performed in symptomatic patients, the prevalence of these events could be even higher if studied in a prospective way with active TEE

screening in all patients.

We found a higher prevalence of venous TEE (87.5\%) and most of the events were related to the deep venous system of the pelvis and lower limbs. These data are comparable with the literature, which indicates the involvement of the lower limbs, pulmonary system, portal and mesenteric as main locations for TEE in IBD patients. ${ }^{5}$

Most of the patients sought medical attention already showing signs and symptoms of TEE. Thus, we can infer that factors frequently related to thromboembolism such as hospitalization, immobilization and the use of intravenous devices were not the most relevant in our study. ${ }^{5}$ The indications for thromboprophylaxis in outpatients are restricted to: (1) patients who already had TEE when IBD was in moderate to severe activity (in this case for up to 3 months of illness in remission) and (2) to those patients who presented TEE without IBD activity but without clearly identified TEE etiology (in this case indefinitely). ${ }^{7}$

The indication of prophylaxis to all patients with active IBD would not be cost-effective as concluded by Nguyen and Sharma. ${ }^{11}$ In this way it would be of great value to identify a risk factor for TEE with standardized detection methodology which can be routinely used in clinical practice.

We observed a significant association between hypoalbuminemia and TEE in our cohort of patients. Patients with serum albumin of less than $2.95 \mathrm{~g} / \mathrm{dL}$ presented a significant increase in the risk of developing TEE (RR, 1.72; 95\% CI, 1.172.53). The study published by Olson et al., ${ }^{12}$ in a cohort of cere- 
bral ischemic events, also found association between hypoalbuminemia and TEE, in addition to a relationship between inflammation (elevation of CRP) and TEE. This was not detected in our study, as the CRP levels were similar between the groups.

More recently a study evaluating the in vitro influence of serum albumin on hemostasis has evidenced an antithrombotic effect of albumin when found in physiological or elevated serum concentrations. According to the study, in primary hemostasis, albumin would act as an anticoagulant because of its ability to bind to epinephrine exposed in the endothelium, coating tissue and inhibiting platelet deposition. Other mechanisms are described but not fully understood: albumin is able to bind to arachidonic acid, which would cause an inhibitory action on platelet aggregation, since the cytoxigenases derived from platelets would be deprived of its substrate and could not form the platelet agonist thromboxane A2. Albumin may also be capable of inducing, in a manner dependent and proportionally to its concentration, the formation of nitric oxide in macrophages, a potent platelet inhibitor. When bound to prostacyclin (inhibitor of platelet aggregation), albumin prevents its degradation, which would optimize its antiplatelet effect. $^{13-16}$

Albumin seems to be related to fibrinogen. The study of Winstedt et al. ${ }^{16}$ aimed at evaluating the response of patients who developed dilutional coagulopathy after administration of colloids, comparing the natural colloid (albumin) with the synthetic ones, demonstrated that albumin-induced coagulopathy was more effectively reversed after the addition of fibrinogen concentrate. These data may help explain the relationship between hypoalbuminemia and TEE, but it is unclear whether serum albumin is mechanically involved or whether this correlation is a manifestation of an underlying inflammatory processes. ${ }^{14,16}$

Despite the evidence of thromboembolic risk associated with IBD, only in 2008 did the American College of Chest Physicians recommend antithrombotic prophylaxis specifically for IBD patients. ${ }^{17}$ Part of our study contemplates a period prior to the widespread dissemination of this guideline, which could justify the large number of hospitalized patients without antithrombotic prophylaxis.

Our study was the first to demonstrate the importance of albumin as a possible marker of risk for TEE in patients with active IBD. However, it presents limitations that must be considered before interpretation of the results. First, this was performed in a reduced sample of patients, being a retrospective study and coming from a single center, with no sample size calcula- tion, using a convenience sample of patients. Secondly, it was performed in patients without adequate prophylaxis, what could represent a selection bias of the included patients. Prospective studies with wider sample of patients, properly powered to check differences are necessary to corroborate our findings.

In conclusion, the prevalence of TEE identified in admitted patients with active IBD without antithrombotic prophylaxis was significant. In patients with TEE, albumin levels were significantly lower, and hypoalbuminemia was considered a risk factor for the development of TEE in this population. Albumin dosage is a simple and accessible test that could be used to stratify risk for TEE in patients with active IBD, especially in outpatient treatment.

\section{FINANCIAL SUPPORT}

The authors received no financial support for the research, authorship, and/or publication of this article.

\section{CONFLICT OF INTEREST}

No potential conflict of interest relevant to this article was reported.

\section{AUTHOR CONTRIBUTION}

Methodology: Imbrizi MR, Kotze PG, Montes CG, de Souza Almeida JR, and Ribeiro Cabral VL. Statistical analysis: Magro DO. Research: Imbrizi MR, Secundo TML, and Cunha-Silva M. Writing: Imbrizi MR and Magro DO. Writing-review and editing: Kotze PG and Ribeiro Cabral VL. Approval of final manuscript: all authors.

\section{REFERENCES}

1. Giannotta M, Tapete G, Emmi G, Silvestri E, Milla M. Thrombosis in inflammatory bowel diseases: what's the link? Thromb J 2015;13:14.

2. Bargen JA, Barker NW. Extensive arterial and venous thrombosis complicating chronic ulcerative colitis. Arch Intern Med 1936;58:17-31.

3. Miehsler W, Reinisch W, Valic E, et al. Is inflammatory bowel disease an independent and disease specific risk factor for thromboembolism? Gut 2004;53:542-548.

4. Irving PM, Pasi KJ, Rampton DS. Thrombosis and inflamma- 
tory bowel disease. Clin Gastroenterol Hepatol 2005;3:617628.

5. Papa A, Gerardi V, Marzo M, Felice C, Rapaccini GL, Gasbarrini A. Venous thromboembolism in patients with inflammatory bowel disease: focus on prevention and treatment. World J Gastroenterol 2014;20:3173-3179.

6. Silverberg MS, Satsangi J, Ahmad T, et al. Toward an integrated clinical, molecular and serological classification of inflammatory bowel disease: report of a Working Party of the 2005 Montreal World Congress of Gastroenterology. Can J Gastroenterol 2005;19 Suppl A:5A-36A.

7. Nguyen GC, Bernstein CN, Bitton A, et al. Consensus statements on the risk, prevention, and treatment of venous thromboembolism in inflammatory bowel disease: Canadian Association of Gastroenterology. Gastroenterology 2014;146:835848.

8. Harvey RF, Bradshaw JM. A simple index of Crohn's-disease activity. Lancet 1980;1:514.

9. Truelove SC, Witts LJ. Cortisone in ulcerative colitis; final report on a therapeutic trial. Br Med J 1955;2:1041-1048.

10. Physical status: the use and interpretation of anthropometry. Report of a WHO Expert Committee. World Health Organ Tech Rep Ser 1995;854:1-452.

11. Nguyen GC, Sharma S. Feasibility of venous thromboembo- lism prophylaxis during inflammatory bowel disease flares in the outpatient setting: a decision analysis. Inflamm Bowel Dis 2013;19:2182-2189.

12. Olson NC, Cushman M, Lutsey PL, et al. Inflammation markers and incident venous thromboembolism: the Reasons for Geographic And Racial Differences in Stroke (REGARDS) cohort. J Thromb Haemost 2014;12:1993-2001.

13. Paar M, Rossmann C, Nusshold C, et al. Anticoagulant action of low, physiologic, and high albumin levels in whole blood. PLoS One 2017;12:e0182997.

14. Jørgensen KA, Stoffersen E. On the inhibitory effect of albumin on platelet aggregation. Thromb Res 1980;17:13-18.

15. Folsom AR, Lutsey PL, Heckbert SR, Cushman M. Serum albumin and risk of venous thromboembolism. Thromb Haemost 2010;104:100-104.

16. Winstedt D, Hanna J, Schött U. Albumin-induced coagulopathy is less severe and more effectively reversed with fibrinogen concentrate than is synthetic colloid-induced coagulopathy. Scand J Clin Lab Invest 2013;73:161-169.

17. Geerts WH, Bergqvist D, Pineo GF, et al. Prevention of venous thromboembolism: American College of Chest Physicians Evidence-Based Clinical Practice Guidelines (8th Edition). Chest 2008;133(6 Suppl):381S-453S. 